\title{
Automated Differential Diagnosis of Early Parkinsonism Using Metabolic Brain Networks: A Validation Study
}

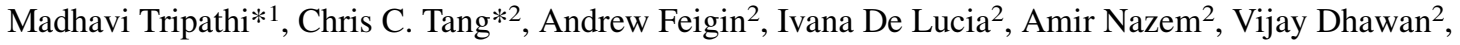 \\ and David Eidelberg ${ }^{2}$ \\ ${ }^{1}$ Department of Nuclear Medicine \& PET, All India Institute of Medical Sciences, New Delhi, India; and ${ }^{2}$ Center for Neurosciences, \\ The Feinstein Institute for Medical Research, Manhasset, New York
}

The differentiation of idiopathic Parkinson disease (IPD) from multiple system atrophy (MSA) and progressive supranuclear palsy (PSP), the most common atypical parkinsonian look-alike syndromes (APS), can be clinically challenging. In these disorders, diagnostic inaccuracy is more frequent early in the clinical course when signs and symptoms are mild. Diagnostic inaccuracy may be particularly relevant in trials of potential disease-modifying agents, which typically involve participants with early clinical manifestations. In an initial study, we developed a probabilistic algorithm to classify subjects with clinical parkinsonism but uncertain diagnosis based on the expression of metabolic covariance patterns for IPD, MSA, and PSP. Classifications based on this algorithm agreed closely with final clinical diagnosis. Nonetheless, blinded prospective validation is required before routine use of the algorithm can be considered. Methods: We used metabolic imaging to study an independent cohort of 129 parkinsonian subjects with uncertain diagnosis; $77(60 \%)$ had symptoms for $2 y$ or less at the time of imaging. After imaging, subjects were followed by blinded movement disorders specialists for an average of $2.2 \mathrm{y}$ before final diagnosis was made. When the algorithm was applied to the individual scan data, the probabilities of IPD, MSA, and PSP were computed and used to classify each of the subjects. The resulting image-based classifications were then compared with the final clinical diagnosis. Results: IPD subjects were distinguished from APS with $94 \%$ specificity and 96\% positive predictive value (PPV) using the original 2-level logistic classification algorithm. The algorithm achieved $90 \%$ specificity and $85 \%$ PPV for MSA and 94\% specificity and $94 \%$ PPV for PSP. The diagnostic accuracy was similarly high (specificity and PPV $>90 \%$ ) for parkinsonian subjects with short symptom duration. In addition, 25 subjects were classified as level I indeterminate parkinsonism and 4 more subjects as level II indeterminate APS. Conclusion: Automated pattern-based image classification can improve the diagnostic accuracy in patients with parkinsonism, even at early disease stages.

Key Words: Parkinson disease; differential diagnosis; brain networks; glucose metabolism; automated classification algorithm

J Nucl Med 2016; 57:60-66

DOI: 10.2967/jnumed.115.161992

Received Jun. 15, 2015; revision accepted Oct. 1, 2015.

For correspondence or reprints contact: David Eidelberg, Center for Neurosciences, The Feinstein Institute for Medical Research, 350 Community Dr., Manhasset, NY 11030.

E-mail: david1@nshs.edu

${ }^{*}$ Contributed equally to this work.

Published online Oct. 8, 2015.

COPYRIGHT (C 2016 by the Society of Nuclear Medicine and Molecular Imaging, Inc.
A ccurate early diagnosis of idiopathic Parkinson disease (IPD) remains a clinical challenge. Motor signs of parkinsonism, particularly akinesia and rigidity, feature prominently in the presentation of IPD as well as atypical parkinsonian look-alike syndromes (APS), including multiple system atrophy (MSA) and progressive supranuclear palsy (PSP). As a result, up to $35 \%$ of patients with parkinsonism are initially misdiagnosed (1) and only $53 \%$ accuracy is found in the clinical diagnosis of early IPD patients responsive to medication (2). The accurate differential diagnosis of individual subjects in this population is critical for several reasons. IPD and APS differ with respect to the natural history of the illness and the response to antiparkinsonian treatment (3). Misdiagnosis can, therefore, be a relevant consideration in the design of clinical trials to assess new therapies, particularly those directed at early, untreated patients (4).

Several structural MR imaging techniques have been used to discriminate individuals with IPD from APS based on differences in tissue signal or regional volume loss $(5,6)$. Radionuclide imaging with PET and SPECT in conjunction with dopaminergic tracers has also been proposed for this purpose $(7,8)$. The differential diagnosis of parkinsonian disorders based on these methods has generally relied on the analysis of imaging signal from single brain regions, most notably the basal ganglia. Given that the basal ganglia are involved in both IPD and APS, measurements of anatomic or functional change in these regions may not be specific enough for an accurate differential diagnosis at the individual case level.

An alternative imaging approach for the differential diagnosis of parkinsonian movement disorders involves spatial covariance mapping, a form of pattern analysis (9-11). Neurodegenerative disorders such as IPD, MSA, and PSP are characterized by stereotyped patterns of regional change on postmortem examination. Analogously, in living patients, these illnesses are associated with characteristic disease-related patterns of abnormal cerebral function identified in the resting state $(9,12,13)$. Given the high reproducibility of diseaserelated topographies across individual subjects and populations $(9,12,14,15)$, quantitative indices of pattern expression can be used as a functional descriptor of disease progression at the network level in individual subjects $(11,16,17)$.

Along these lines, we explored the possibility of using subject expression values for multiple disease-related patterns in concert to categorize individual patients based on their scans. As a proof of principle, we developed a logistic regression algorithm based on computed subject scores for previously validated metabolic covariance patterns for IPD (Parkinson disease-related pattern [PDRP] $(9,14,18,19)$ ), MSA (multiple system atrophy-related pattern 
[MSARP] $(20,21)$ ), and PSP (progressive supranuclear palsy-related pattern [PSPRP] $(20,22)$ ) to classify a North American training cohort of 167 individuals with parkinsonism with an inconclusive clinical diagnosis. These subjects underwent metabolic brain imaging an average of $2.6 \mathrm{y}$ before a final clinical diagnosis was made. The pattern-based probabilistic algorithm provided high specificity (94\%-97\%) in discriminating IPD from APS as well as MSA from PSP. Leave-one-out cross validation was applied to these data revealing a high level of replicability for this diagnostic algorithm (11). Even so, the clinical utility of this approach could not be considered further without validation in an independent testing cohort. In the current study, we applied the original logistic algorithm to metabolic scans from an Indian testing cohort of 129 parkinsonian subjects with uncertain clinical diagnosis who were followed by movement disorders experts blinded to the imaging results. The primary objective of this study was to prospectively validate the original logistic classification algorithm, previously developed from the North American cohort (11), in the new Indian cohort. In addition, because the greatest clinical challenge exists in patients with relatively recent symptom onset, subjects with relatively short disease duration $(\leq 2 \mathrm{y})$ were preferentially chosen as participants and separately analyzed in this validation cohort.

\section{MATERIALS AND METHODS}

\section{Patients}

One hundred sixty-five parkinsonian subjects with uncertain clinical diagnoses were referred for diagnostic ${ }^{18} \mathrm{~F}$-FDG PET between November 2008 and January 2011 (Fig. 1). Of these, 36 subjects were excluded because of structural abnormalities on routine MR imaging $(n=10$ : 3 with basal ganglia ischemia, 2 with hydrocephalus, and 5 with severe brain atrophy), alternative final clinical diagnosis ( $n=4: 1$ with dementia with Lewy bodies, 1 with normal pressure hydrocephalus, and 2 with frontotemporal dementia/progressive nonfluent aphasia), and lack of final clinical diagnosis due to inadequate follow-up $(n=22)$.

The remaining 129 subjects ( 90 men and 39 women; mean age \pm SD, $56.2 \pm 10.6$ y; motor Unified Parkinson Disease Rating Scale [UPDRS], $25.1 \pm 9.4$; symptom duration, $2.7 \pm 1.5 \mathrm{y}$ ) underwent ${ }^{18} \mathrm{~F}$-FDG PET at the Institute of Nuclear Medicine and Allied Sciences (INMAS). Of these, $77(60 \%)$ subjects (54 men and 23 women; mean age \pm SD, $56.8 \pm 9.7$ y) had symptom duration of $2 \mathrm{y}$ or less and comprised a short-duration subgroup that was separately analyzed. In each of the

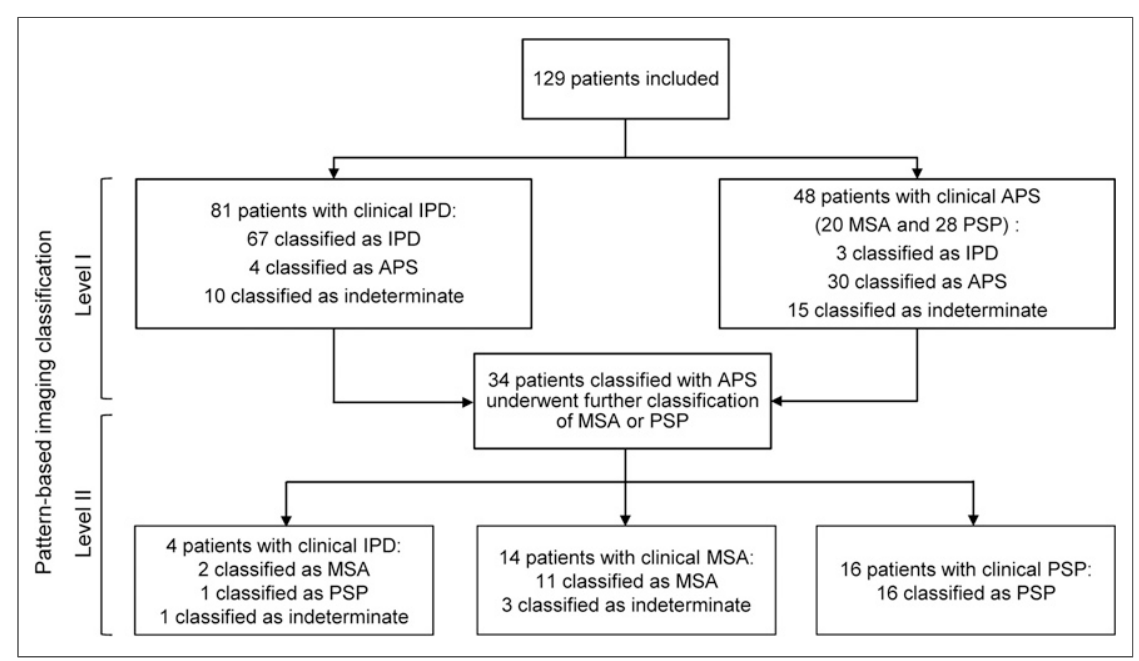

FIGURE 1. Study design and pattern-based classification procedure. subjects, the final clinical diagnosis was made by a movement disorders specialist after at least 2 office visits. The mean time interval between imaging and final diagnosis was $2.2 \pm 0.4 \mathrm{y}$ after imaging. In all cases, the diagnosis was based on consensus criteria for the diagnosis of IPD (United Kingdom Brain Bank Criteria (23)), MSA (24), and PSP (25) and was confirmed by a second movement disorders specialist. Both clinical experts were blinded to the imaging findings at the time of final clinical diagnosis. Because of the excellent diagnostic agreement between the 2 specialists (Cohen's $\kappa=0.91$; 95\% confidence interval, 0.84-0.98) and the close concordance with postmortem data reported for movement disorders experts after 2 y of clinical follow-up (26), we chose the final clinical diagnosis as the endpoint for the current study.

The study was approved by the ethical committee at INMAS, and written informed consent was obtained from each patient after a detailed explanation of the procedure.

\section{Imaging and Preprocessing}

${ }^{18} \mathrm{~F}$-FDG PET was performed on patients after a 12-h fast. Antiparkinsonian medications were withheld for at least $12 \mathrm{~h}$ before imaging. Subjects received $185-296 \mathrm{MBq}(5-8 \mathrm{mCi})$ of ${ }^{18} \mathrm{~F}-\mathrm{FDG}$ by intravenous injection with eyes open in a silent, dimly lit room. Scanning was performed on the Discovery STE16 PET tomograph (GE Healthcare) at INMAS. This camera has a transaxial resolution of $5.12 \mathrm{~mm}$ in full width at half maximum in 3-dimensional mode at an offset of $1 \mathrm{~cm}$ from the center of the field of view. An initial scout film of the head was obtained, followed by low-dose CT for attenuation correction and coregistration. PET imaging was begun $60 \mathrm{~min}$ after injection; a single bed 3-dimensional emission scan was acquired for $20 \mathrm{~min}$ for each subject.

To ensure the compatibility in this validation study, we used the same protocols as used in our proof-of-principle study for all procedures of image preprocessing and network computations (11). Specifically, image preprocessing was performed using statistical parametric mapping software (Wellcome Department of Cognitive Neurology), running on Matlab (The MathWorks Inc.). ${ }^{18}$ F-FDG PET images were spatially normalized into a standard brain space of the International Consortium for Brain Mapping (27) and smoothed with a $10-\mathrm{mm}$ gaussian filter to increase the signal-to-noise ratio, as described previously (28).

\section{Network Computations}

Expression values (subject scores) for each of the 3 relevant previously validated disease-related metabolic covariance patterns (PDRP, MSARP, and PSPRP) were computed on a prospective individual-scan basis as described elsewhere $(10,13)$ using existing software (ScAnVp, freely available at http:// www.feinsteinneuroscience.org/). Subject scores for each disease pattern were standardized $(z-$ scored) with respect to corresponding values from the same group of 42 healthy volunteer subjects (mean age $\pm \mathrm{SD}, 51.6 \pm 14.6 \mathrm{y}$ ) used for reference in the original proof-of-principle study (11). As before, subject scores for each pattern were standardized such that the mean expression value for the reference sample was zero, with an SD of 1.

\section{Differential Diagnosis}

We applied the previously developed logistic classification algorithm (11) to the PDRP, MSARP, and PSPRP subject scores from each of the participants of the Indian testing cohort.

The original logistic models for classification of IPD, MSA, and PSP derived from the North American training cohort (11) are described in Supplemental Table 1 (supplemental 
materials are available at http://jnm.snmjournals.org). Prospective application of these models allowed us to calculate the probability of each disease for individual subjects of the current cohort. On the basis of these probabilities, we classified each of the subjects according to a 2-level procedure, as previously reported (11). At level I, each subject was classified as IPD or APS, or as indeterminate parkinsonism, by comparing the subject's probabilities to the cutoff probabilities for IPD (0.81) and APS (0.79) determined in the original study (11). Patients who had a higher probability than the cutoff value for IPD were classified as IPD, whereas those with a higher probability than the cutoff value for APS were classified as APS. Subjects with probabilities lower than the cutoff values of both IPD and APS were classified as indeterminate parkinsonism. At level II, subjects classified at level I as APS were further subclassified as MSA or PSP, or as indeterminate APS, using the previously reported cutoff probabilities for MSA (0.74) and PSP (0.55) (11). Likewise, subjects with a higher probability than the cutoff for either of the 2 major APS subtypes were classified as MSA or PSP. Subjects with probabilities lower than the cutoff values for the 2 conditions were classified as indeterminate APS.

Image-based classification was performed in each subject without knowledge of the final clinical diagnosis. On the basis of the classification results of individual subjects, discriminative measures (sensitivity, specificity, positive predictive value [PPV], and negative predictive value [NPV]) for the analysis at each level (level I, PD and APS; level II, MSA and PSP) were computed separately for the whole group $(n=129)$ and for the short-duration sample $(n=77)$. At each level, indeterminate cases were included as misclassified subjects in the calculation of discriminative measures. Because level I indeterminate cases had a similar likelihood ( $\sim 50 \%)$ of having IPD or APS, these subjects were not further classified as MSA or PSP at level II (11). Thus, as implemented in the original logistic algorithm, level I indeterminate cases in the present cohort were excluded from the level II analysis and the calculation of discriminative measures at this level. Moreover, because the proportion of APS patients in this Indian cohort (37\%) was substantially higher than the expected prevalence in the general population (29), the PPVs reported in this study were features of the current sample, which was representative of the patients in the referring tertiary movement disorders clinics. In addition, receiver-operating characteristic (ROC) analysis was conducted for each disease condition using the entire dataset and was then limited to the subgroup of short-duration ( $\leq 2 \mathrm{y})$ cases.

Logistic regression analysis was performed in SAS 9.3 (SAS Institute Inc.), and other statistical tests were performed in SPSS 14.0 (SPSS Inc.). All tests were considered significant for a $P$ level of less than 0.05 .

\section{RESULTS}

\section{Analysis of Whole Group}

The demographic features of the subjects are summarized in Table 1. Among the 129 subjects with uncertain parkinsonism that were included, 81 were subsequently diagnosed clinically as having IPD, and all of them were responsive to levodopa treatment. Of the remaining subjects, 20 were diagnosed with MSA and 28 with PSP. The average disease duration was $2.7 \pm 1.5 \mathrm{y}$ at the time of imaging; a final diagnosis was made an average of $2.2 \pm 0.4 \mathrm{y}$ after the imaging procedure.

PDRP, MSARP, and PSPRP expression values for the subjects, grouped by final clinical diagnosis, are displayed in Figure 2 . The resulting subject scores were used in conjunction with the previously reported logistic algorithm to compute the probabilities of IPD and APS in each of the subjects. We then compared the image-based classification with their final clinical diagnosis. Of the 129 patients, 70 (67 were diagnosed clinically as IPD, 1 as MSA, and 2 as PSP) were classified as having IPD, whereas 34 (4 were subsequently diagnosed clinically as IPD, 14 as MSA, and 16 as
PSP) were classified as having APS. The remaining 25 subjects (10 IPD, 5 MSA, and 10 PSP), accounting for $19 \%$ of the total, did not satisfy the prespecified cutpoint criteria for classification as either IPD or APS (Fig. 3A, left) and were therefore categorized as indeterminate parkinsonism. Overall, the first-level analysis (Table 2) resulted in $83 \%$ sensitivity, $94 \%$ specificity, $96 \% \mathrm{PPV}$, and $76 \%$ NPV for the classification of IPD, and $63 \%$ sensitivity, $95 \%$ specificity, $88 \%$ PPV, and $81 \%$ NPV for the classification of APS (Table 2). The area under the curve (AUC) for the ROC analyses of the whole sample (Fig. 3A, right) was $0.95(P<0.0001)$ for both IPD and APS.

The 34 subjects classified as APS at level I underwent secondlevel analysis (Figs. 3B and 3C, left) to further differentiate between MSA and PSP. This analysis (Table 2) resulted in $79 \%$ sensitivity, 90\% specificity, $85 \%$ PPV, and $86 \%$ NPV for the classification of MSA, and $100 \%$ sensitivity, $94 \%$ specificity, $94 \%$ PPV, and 100\% NPV for the classification of PSP. ROC analysis (Figs. 3B and 3C, right) revealed a significant AUC of 0.93 for MSA $(P<0.0001)$ and 0.99 for PSP $(P<0.0001)$. In addition, 4 (12\%; 1 IPD and 3 MSA) of the 34 subjects did not satisfy the prespecified classification criteria for either MSA or PSP and were categorized as indeterminate APS at the second level.

\section{Analysis of Short-Duration Subgroup}

Seventy-seven subjects (40 IPD, 16 MSA, and 21 PSP), accounting for $60 \%$ of the total, had symptoms of short duration ( $\leq 2 \mathrm{y}$ ). For this subgroup, the average duration of symptoms at the time of imaging was $1.7 \pm 0.4 \mathrm{y}$. The final clinical diagnosis in these subjects was made $2.2 \pm 0.5 \mathrm{y}$ after imaging. On the basis of the classification algorithm, 38 of these early stage subjects were categorized as IPD (36 were subsequently diagnosed clinically as IPD, 1 as MSA, and 1 as PSP), whereas 24 were classified as APS (1 clinically diagnosed as IPD, 11 as MSA, and 12 as PSP). The remaining 15 (19\%) subjects (3 subsequently classified clinically as IPD, 4 as MSA, and 8 as PSP) were categorized by the algorithm (Fig. 4A, left) as indeterminate parkinsonism. Thus, for this early subgroup, the first-level analysis (Table 2) resulted in $90 \%$ sensitivity, 95\% specificity, 95\% PPV, and $90 \%$ NPV for IPD, and $62 \%$ sensitivity, $98 \%$ specificity, $96 \%$ PPV, and 74\% NPV for APS. ROC analysis (Fig. 4A, right) revealed an AUC of $0.96(P<0.0001)$ for both IPD and APS at the first level.

Second-level analysis for the 24 subjects (Figs. 4B and 4C, left) who were classified as APS (Table 2) resulted in $73 \%$ sensitivity, $92 \%$ specificity, $89 \% \mathrm{PPV}$, and $80 \% \mathrm{NPV}$ for MSA, and $100 \%$ sensitivity, $100 \%$ specificity, $100 \%$ PPV, and $100 \%$ NPV for PSP. ROC analysis (Figs. 4B and 4C, right) showed an AUC of 0.94 $(P<0.0001)$ for MSA and 1.00 for PSP $(P<0.0001)$ at the second level. Three (13\%; 3 MSA) of the 24 subjects were categorized as indeterminate APS.

\section{Analysis of Cases with Atrophy}

To examine the potential confounding effect of brain atrophy on the classification of individual subjects, we additionally applied the algorithm to PET data from the 5 subjects who were excluded from the primary analysis because of atrophy. These individuals were all subsequently diagnosed with APS on clinical grounds: 2 with MSA and 3 with PSP. Of those with MSA, one subject was classified by the algorithm as indeterminate parkinsonism at level I and the other as indeterminate APS at level II. Of the PSP cases, all were classified as level I indeterminate parkinsonism. 
TABLE 1

Demographic Characteristics

\begin{tabular}{|c|c|c|c|}
\hline \multirow[b]{2}{*}{ Characteristic } & \multirow[b]{2}{*}{ All patients $(n=129)$} & \multicolumn{2}{|c|}{ Symptom duration (y) } \\
\hline & & $\leq 2(n=77)$ & $>2(n=52)$ \\
\hline \multicolumn{4}{|l|}{ IPD } \\
\hline No. of patients & 81 & 40 & 41 \\
\hline \multicolumn{4}{|l|}{ Sex } \\
\hline Male & 59 & 31 & 28 \\
\hline Female & 22 & 9 & 13 \\
\hline Age at ${ }^{18} \mathrm{~F}-\mathrm{FDG}$ PET $(\mathrm{y})$ & $53.5(10.8)^{\star}$ & $53.7(9.9)^{\star}$ & $53.4(11.7)$ \\
\hline Symptom duration at ${ }^{18} \mathrm{~F}-\mathrm{FDG}$ PET (y) & $2.9(1.8)^{\star}$ & $1.6(0.5)$ & $4.2(1.6)$ \\
\hline Hoehn and Yahr stage & $2.5(2.8)$ & $1.7(0.5)^{\star}$ & $3.3(3.7)$ \\
\hline UPDRS motor & $23.7(10.5)^{\star}$ & $19.5(7.1)^{\star}$ & $27.8(11.7)$ \\
\hline Clinical follow-up (y) & $2.3(0.5)^{\star}$ & $2.4(0.6)^{\star}$ & $2.1(0.3)$ \\
\hline \multicolumn{4}{|l|}{ MSA } \\
\hline No. of patients & 20 & 16 & 4 \\
\hline \multicolumn{4}{|l|}{ Sex } \\
\hline Male & 15 & 11 & 4 \\
\hline Female & 5 & 5 & 0 \\
\hline Age at ${ }^{18}$ F-FDG PET (y) & $58.0(9.4)$ & $57.4(8.8)$ & $60.5(12.5)$ \\
\hline Symptom duration at ${ }^{18} \mathrm{~F}-\mathrm{FDG}$ PET (y) & $2.2(0.8)$ & $1.8(0.3)$ & $3.5(0.6)$ \\
\hline Hoehn and Yahr stage & $2.7(0.6)$ & $2.6(0.5)$ & $3.1(0.6)$ \\
\hline UPDRS motor & $24.9(6.7)$ & $23.6(5.5)$ & $30.3(9.5)$ \\
\hline Clinical follow-up (y) & $1.9(0.1)$ & $2.0(0.1)$ & $1.8(0.2)$ \\
\hline \multicolumn{4}{|l|}{ PSP } \\
\hline No. of patients & 28 & 21 & 7 \\
\hline \multicolumn{4}{|l|}{ Sex } \\
\hline Male & 16 & 12 & 4 \\
\hline Female & 12 & 9 & 3 \\
\hline Age at ${ }^{18}$ F-FDG PET (y) & $62.5(7.6)$ & $62.2(7.8)$ & $63.6(7.3)$ \\
\hline Symptom duration at ${ }^{18} \mathrm{~F}-\mathrm{FDG}$ PET (y) & $2.3(1.1)$ & $1.8(0.4)$ & $3.9(1.1)$ \\
\hline Hoehn and Yahr stage & $2.8(0.6)$ & $2.6(0.6)$ & $3.1(0.4)$ \\
\hline UPDRS motor & $29.3(5.8)$ & $27.8(5.7)$ & $33.7(3.7)$ \\
\hline Clinical follow-up (y) & $2.0(0.2)$ & $2.0(0.1)$ & $2.1(0.4)$ \\
\hline \multicolumn{4}{|c|}{$\begin{array}{l}\text { *Denotes differences }(P<0.05) \text { across the } 3 \text { disease groups by } 1 \text {-way ANOVA. } \\
\text { Data are mean, with SD in parentheses. } \\
\text { UPDRS = Unified Parkinson Disease Rating Scale. }\end{array}$} \\
\hline
\end{tabular}

\section{DISCUSSION}

In this study, we used the original network-based logistic algorithm (11) to classify individuals in an independent testing sample based on their metabolic scans. In accordance with the earlier findings (11), this automated image classification approach resulted in accurate differential diagnosis, with excellent discrimination of IPD from APS (level I) and MSA from PSP (level II) at the individual subject level. The data overall demonstrate the potential utilities of the algorithm in improving the recruitment of participants for clinical trials and in assisting clinicians to make a more accurate diagnosis for patients with early stage disease.

Specifically, we applied the published classification algorithm to scan data from the Indian population using the identical disease-related covariance patterns and the criteria for probabilistic classification that were used in the previously published North American training cohort (11). Single-case classification according to this approach resulted in comparably high diagnostic accuracy for IPD in the 2 populations (training: $97 \%$ specificity, $98 \%$ PPV; testing: $94 \%$ specificity, 96\% PPV). Likewise, image-based classification of APS was also diagnostically accurate in both the North American and the Indian samples (training: 98\% specificity, 97\% PPV; testing: $95 \%$ specificity, $88 \%$ PPV). In addition, there was no significant difference in the percentage of indeterminate cases in the Indian and North American samples at the 2 levels of classification analyses (level I: $19 \%$ vs. $14 \%, P=0.25$; level II: $12 \%$ vs. $12 \%, P=$ $0.99 ; \chi^{2}$ tests). In both samples, we found that at final diagnosis, 


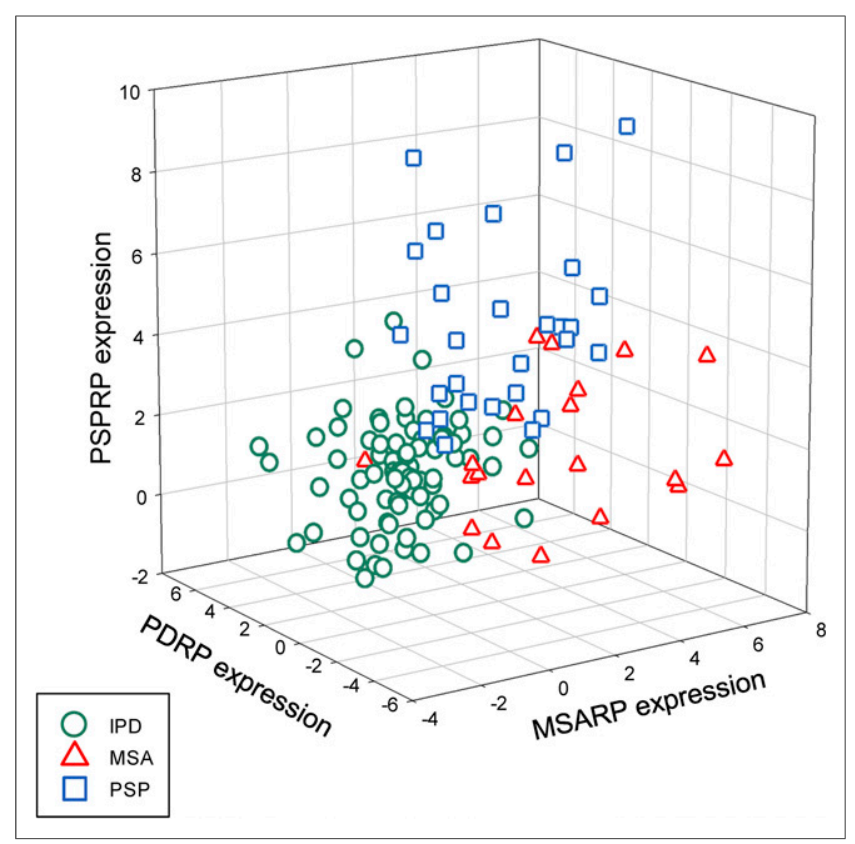

FIGURE 2. Three-dimensional plot of pattern expression. Expression values for PDRP ( $x$-axis), MSARP ( $y$-axis), and PSPRP ( $z$-axis) topographies are shown for Indian validation cohort. This group comprised 129 parkinsonian patients with uncertain clinical diagnosis. On the basis of serial clinical examinations by movement disorders specialists blind to scan data, 81 of these subjects were subsequently diagnosed with IPD, 20 with MSA, and 28 with PSP. Pattern expression values were computed in ${ }^{18} \mathrm{~F}-\mathrm{FDG}$ PET scans from these subjects acquired $2.2 \pm 0.4 \mathrm{y}$ (mean $\pm \mathrm{SD}$ ) before final clinical diagnosis.

indeterminate cases had similar likelihoods of approximately $50 \%$ of being classified clinically as IPD or APS (level I) and MSA or PSP (level II) (11). Thus, indeterminate cases may represent the clinically more challenging patients who can benefit greatly from continued clinical follow-up and repeated imaging as the underlying disease progresses. Moreover, such cases, if identified, may be excluded from recruitment to help improve the efficiency and reduce the cost of clinical trials.

An important feature of the current study was the greater percentage of subjects in the Indian testing cohort with short ( $\leq 2 \mathrm{y}$ ) symptom duration than in the original North American training sample $(77 / 129=60 \%$ vs. $55 / 167=33 \%$, respectively). Indeed, for the whole group, symptom duration at the time of imaging was substantially shorter for the current testing sample relative to the original training dataset (New Delhi, $2.7 \pm 1.5 \mathrm{y}$; Manhasset, $5.0 \pm 3.8 \mathrm{y}, P<0.0001$; Student $t$ test). The specificity and PPV for pattern-based classification of individual subjects at level I (IPD vs. APS) was high ( $\geq 95 \%$ ) even for the short-duration cases (with $\leq 2$ y of symptoms). Thus, the imaging-based classifications accorded well with the clinical gold standard diagnosis reached independently by the expert clinicians approximately $2 \mathrm{y}$ after the imaging procedure. Interestingly, sensitivity at level I was higher for IPD than for APS (90\% vs. 62\%), suggesting a greater rate of false-negatives for classifying APS patients with short symptom duration. Among the 15 short-duration subjects (19\%) categorized as indeterminate parkinsonism at level I, 12 were later clinically diagnosed as MSA or PSP and were most of the false-negatives for APS, resulting in a lower sensitivity. Therefore, whereas a subject with indeterminate parkinsonism (level I) drawn at random from the whole sample has similar odds of developing APS or IPD, a comparable subject with short disease duration is 4 times more likely to develop APS as opposed to IPD. Level II analysis of the early APS subjects revealed that the specificity and PPV were high for both MSA ( $92 \%$ and $89 \%$, respectively) and PSP (100\% and $100 \%$, respectively), and the sensitivity of MSA and PSP $(73 \%$ and $100 \%)$ was higher than that reported $(<60 \%)$ for the initial clinical diagnosis of each of these conditions (30,31). Moreover, the percentage of indeterminate cases at both level I and level II were similar for Indian and North American short-duration cases (level I: $19 \%$ vs. $20 \%, P=0.94$; level II: $13 \%$ vs. $11 \%$,

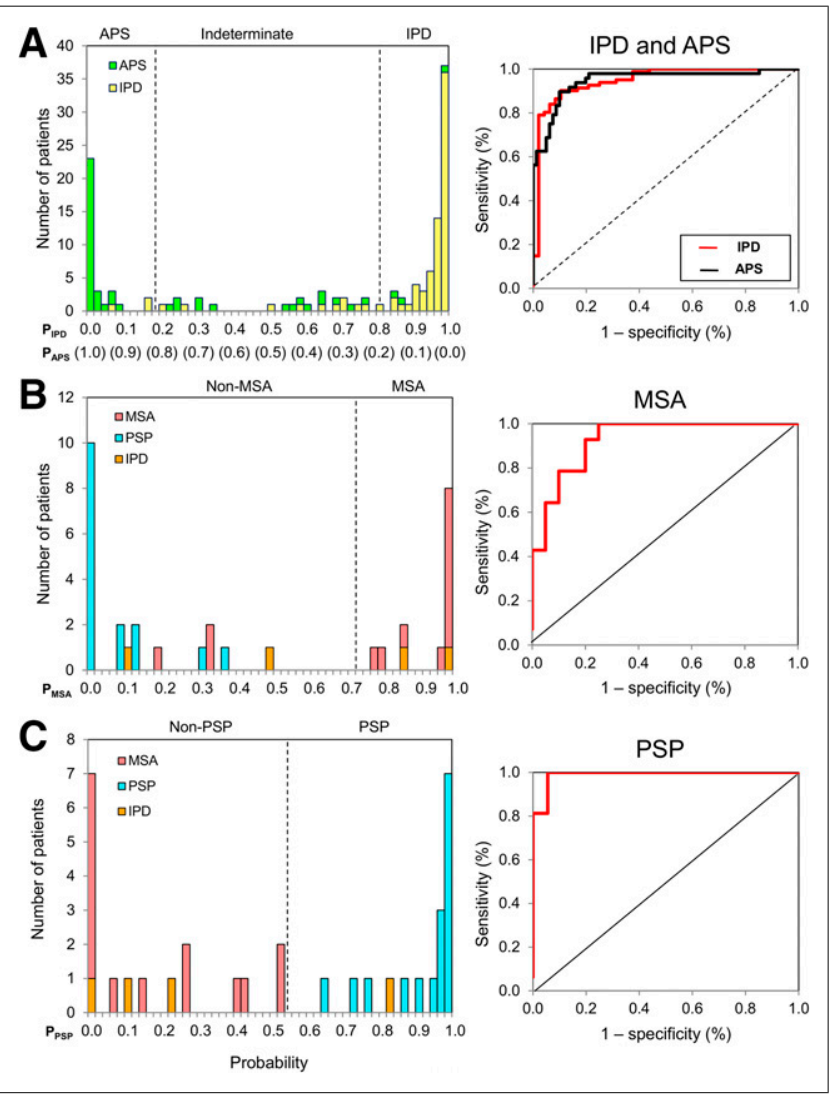

FIGURE 3. Predicted disease probabilities for differential diagnosis of patients with uncertain parkinsonism: whole sample. (A, left) Frequency distribution of predicted probabilities for IPD ( $P_{\text {IPD }}$, top row of $x$-axis) and APS ( $\mathrm{P}_{\text {APS }}$, bottom row of $x$-axis) for current parkinsonian patient sample $(n=129)$. This group comprised 81 subjects who were diagnosed clinically with IPD (yellow bars) and 48 subjects who were diagnosed with APS (green bars). Right and left dashed lines, respectively, denote cutoff probabilities for IPD $\left(P_{I P D}=0.81\right)$ and APS $\left(P_{\text {APS }}=0.79\right)$ determined in our previous study (11). Subjects falling between 2 dashed lines were categorized as indeterminate. (A, right) Display of ROC curves for IPD (red) and APS (black). AUC was high $(\geq 0.95, P<0.0001)$, denoting excellent diagnostic accuracy for the 2 conditions based on logistic discrimination function identified in North American training set (11). (B and C, left) Frequency distributions of predicted probabilities for MSA ( $P_{M S A}, x$-axis in $\left.B\right)$ and PSP ( $\mathrm{P}_{\mathrm{PSP}}, x$-axis in $\mathrm{C}$ ) in 34 subjects who were classified as APS in first-level analysis. This group included 14 patients clinically diagnosed as having MSA (pink bars), 16 with PSP (blue bars), and 4 with IPD (orange bars). (B and $\mathrm{C}$, right) ROC curves for MSA (AUC $=0.93, P<0.0001$ ) (B) and PSP (AUC $=0.99, P<0.0001)(C)$ were consistent with excellent diagnostic accuracy for 2 atypical parkinsonian variant conditions based on original logistic discrimination function (11). 
$P=0.84 ; \chi^{2}$ tests). Overall, these results substantiate the findings of the early patients in the original training sample (11).

The presence of substantial volume loss in early parkinsonism is suggestive of an atypical syndrome $(32,33)$. To mitigate this potential confound, evidence of atrophy on structural imaging was adopted as an exclusion criterion in our study. In addition to confirming the accuracy of the classification algorithm in parkinsonian patients with minimal or no atrophy (11), the current findings provide information on the impact of severe volume loss on pattern-based categorization. We found that all 5 APS patients with severe atrophy at the time of imaging were categorized as indeterminate by the PET-based logistic algorithm. This degree of volume loss was encountered in fewer than $3 \%$ of the referred cases. That said, the inclusion of such subjects may lead to systematic underestimation of the accuracy of this approach. It is not currently known whether diagnostic specificity can be enhanced appreciably by combining MR imaging volume loss measurements with metabolic pattern analysis.

MSA and PSP account for most ( $>90 \%)$ patients with atypical forms of progressive parkinsonism referred to movement disorders specialty clinics $(26,34)$. Nonetheless, the APS category also includes corticobasal degeneration (CBD). This disorder is less common than the other APS subtypes, with an incidence of 0.6$0.9 / 100,000$, as compared with 3.0/100,000 and 5.3/100,000 for MSA and PSP, respectively $(35,36)$. In a recent study, we used

TABLE 2

Discriminative Measures: Pattern-Based Classification Versus Final Clinical Diagnosis

\begin{tabular}{cccc}
\hline & & \multicolumn{2}{c}{ Symptom duration $(\mathrm{y})$} \\
\cline { 3 - 4 } $\begin{array}{c}\text { Discriminative } \\
\text { measure }\end{array}$ & $\begin{array}{c}\text { All patients } \\
(n=129)\end{array}$ & $\begin{array}{c}\leq 2 \\
(n=77)\end{array}$ & $\begin{array}{c}>2 \\
(n=52)\end{array}$ \\
\hline IPD & & & \\
Sensitivity & $83 \%(67 / 81)$ & $90 \%(36 / 40)$ & $76 \%(31 / 41)$ \\
Specificity & $94 \%(45 / 48)$ & $95 \%(35 / 37)$ & $91 \%(10 / 11)$ \\
\hline PPV & $96 \%(67 / 70)$ & $95 \%(36 / 38)$ & $97 \%(31 / 32)$ \\
\hline NPV & $76 \%(45 / 59)$ & $90 \%(35 / 39)$ & $50 \%(10 / 20)$ \\
\hline APS & & & \\
Sensitivity & $63 \%(30 / 48)$ & $62 \%(23 / 37)$ & $64 \%(7 / 11)$ \\
\hline Specificity & $95 \%(77 / 81)$ & $98 \%(39 / 40)$ & $93 \%(38 / 41)$ \\
PPV & $88 \%(30 / 34)$ & $96 \%(23 / 24)$ & $70 \%(7 / 10)$ \\
\hline NPV & $81 \%(77 / 95)$ & $74 \%(39 / 53)$ & $91 \%(38 / 42)$ \\
\hline MSA & & & - \\
Sensitivity & $79 \%(11 / 14)$ & $73 \%(8 / 11)$ & - \\
Specificity & $90 \%(18 / 20)$ & $92 \%(12 / 13)$ & - \\
PPV & $85 \%(11 / 13)$ & $89 \%(8 / 9)$ & - \\
\hline NPV & $86 \%(18 / 21)$ & $80 \%(12 / 15)$ & - \\
\hline PSP & & & - \\
Sensitivity & $100 \%(16 / 16)$ & $100 \%(12 / 12)$ & - \\
Specificity & $94 \%(17 / 18)$ & $100 \%(12 / 12)$ & - \\
PPV & $94 \%(16 / 17)$ & $100 \%(12 / 12)$ & - \\
NPV & $100 \%(17 / 17)$ & $100 \%(12 / 12)$ & - \\
\hline & & & - \\
\hline
\end{tabular}

Data are $\%$ (no. of subjects).

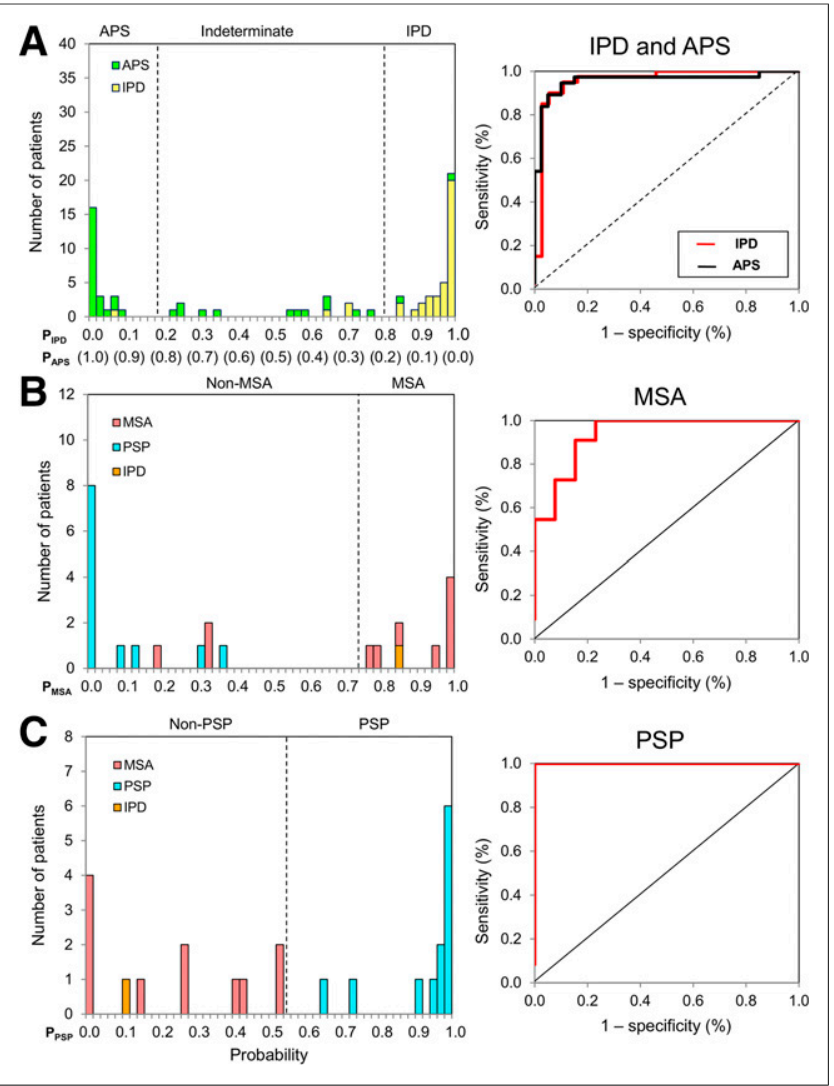

FIGURE 4. Predicted disease probabilities for differential diagnosis of patients with uncertain parkinsonism: subset of early patients. Frequency distribution of predicted probabilities for IPD vs. APS (A), MSA (B), and PSP (C) in 77 members of Indian patient sample (40 IPD, 16 MSA, $21 \mathrm{PSP}$ ) with symptoms of short duration ( $\leq 2 \mathrm{y}$ ) at time of imaging. ROC analysis (IPD and APS: AUC $=0.96, P<0.0001$; MSA: AUC $=$ 0.94, $P<0.0001$; PSP: AUC $=1.00, P<0.0001$ ) disclosed excellent diagnostic accuracy based on logistic discriminant functions identified in original training set (11).

spatial covariance mapping to identify and validate a unique CBDrelated metabolic covariance pattern, termed CBDRP (22). Moreover, prospectively computed expression values for this pattern in conjunction with PSPRP accurately discriminated CBD from PSP in clinically uncertain cases (22). The goal of the present study was to validate the previously reported logistic algorithm for the same diagnostic categories used in its original formulation (11). For that reason, we limited the current APS analysis to MSARP and PSPRP, the 2 disease-related patterns that were used originally to differentiate among the most common progressive forms of parkinsonism. Additional studies will be needed to determine the added value of CBDRP subject scores in discriminating IPD from APS at level I and in differentiating CBD from the other forms of APS at level II.

\section{CONCLUSION}

In this study, we validated the network-based algorithm with metabolic imaging for differential diagnosis of parkinsonian disorders, originally developed from an American patient cohort (11), in an independent cohort of Indian patients with uncertain parkinsonism. In accordance with the previous study, we found that this fully automated approach resulted in accurate image-based 
classification with high specificities and PPVs in differentiating IPD from APS and MSA from PSP on a prospective single-scan basis, especially for early stage patients. Thus, our findings demonstrate potential utility of this algorithm in improving the recruitment of participants for clinical trials and facilitating early diagnosis of parkinsonian syndromes.

\section{DISCLOSURE}

The costs of publication of this article were defrayed in part by the payment of page charges. Therefore, and solely to indicate this fact, this article is hereby marked "advertisement" in accordance with 18 USC section 1734. This work was funded by a fellowship grant from the Indo-US Science and Technology Form and an intramural grant from INMAS. This work was also supported in part by the National Institute of Neurological Disorders and Stroke Morris K. Udall Center of Excellence for Parkinson's Disease Research at The Feinstein Institute for Medical Research (P50 NS071675). The content is solely the responsibility of the authors and does not necessarily represent the official views of the National Institute of Neurological Disorders and Stroke or the National Institutes of Health. The sponsor did not play a role in study design, collection, analysis, and interpretation of data; in writing of the report; or in the decision to submit the paper for publication. David Eidelberg serves on the scientific advisory board and has received honoraria from the Michael J. Fox Foundation for Parkinson's Research; is listed as coinventor of patents regarding markers for use in screening patients for nervous system dysfunction and a method and apparatus for using same, without financial gain; and has received research support from the NIH (NINDS, NIDCD, NIAID) and the Dana Foundation. No other potential conflict of interest relevant to this article was reported.

\section{ACKNOWLEDGMENTS}

We thank Dr. Deepak Gupta for his initial contribution to this study. We also thank Patricia J. Allen for data management and Yoon Young Choi for editorial assistance.

\section{REFERENCES}

1. Tolosa E, Wenning G, Poewe W. The diagnosis of Parkinson's disease. Lancet Neurol. 2006;5:75-86.

2. Adler $\mathrm{CH}$, Beach TG, Hentz JG, et al. Low clinical diagnostic accuracy of early vs advanced Parkinson disease: clinicopathologic study. Neurology. 2014;83: 406-412.

3. Poston KL, Eidelberg D. Functional brain networks and abnormal connectivity in the movement disorders. Neuroimage. 2012;62:2261-2270.

4. Litvan I, Bhatia KP, Burn DJ, et al. Movement Disorders Society Scientific Issues Committee report: SIC Task Force appraisal of clinical diagnostic criteria for Parkinsonian disorders. Mov Disord. 2003;18:467-486.

5. Marquand AF, Filippone M, Ashburner J, et al. Automated, high accuracy classification of Parkinsonian disorders: a pattern recognition approach. PLoS One. 2013;8:e69237.

6. Meijer FJ, van Rumund A, Tuladhar AM, et al. Conventional 3T brain MRI and diffusion tensor imaging in the diagnostic workup of early stage parkinsonism. Neuroradiology. 2015;57:655-669.

7. Park E, Hwang YM, Lee CN, et al. Differential diagnosis of patients with inconclusive parkinsonian features using $\left[{ }^{18} \mathrm{~F}\right] \mathrm{FP}-\mathrm{CIT}$ PET/CT. Nucl Med Mol Imaging. 2014;48:106-113.

8. Suwijn SR, van Boheemen CJ, de Haan RJ, Tissingh G, Booij J, de Bie RM. The diagnostic accuracy of dopamine transporter SPECT imaging to detect nigrostriatal cell loss in patients with Parkinson's disease or clinically uncertain parkinsonism: a systematic review. EJNMMI Res. 2015;5:12.
9. Niethammer M, Eidelberg D. Metabolic brain networks in translational neurology: concepts and applications. Ann Neurol. 2012;72:635-647.

10. Spetsieris P, Ma Y, Peng S, et al. Identification of disease-related spatial covariance patterns using neuroimaging data. J Vis Exp. 2013;76:e50319.

11. Tang CC, Poston K, Eckert T, et al. Differential diagnosis of parkinsonism: a metabolic imaging study using pattern analysis. Lancet Neurol. 2010;9:149158.

12. Eidelberg D. Metabolic brain networks in neurodegenerative disorders: a functional imaging approach. Trends Neurosci. 2009;32:548-557.

13. Spetsieris PG, Eidelberg D. Scaled subprofile modeling of resting state imaging data in Parkinson's disease: methodological issues. Neuroimage. 2011;54:2899-2914.

14. Ma Y, Tang C, Spetsieris P, Dhawan V, Eidelberg D. Abnormal metabolic network activity in Parkinson's disease: test-retest reproducibility. J Cereb Blood Flow Metab. 2007;27:597-605

15. Peng S, Ma Y, Spetsieris PG, et al. Characterization of disease-related covariance topographies with SSMPCA toolbox: effects of spatial normalization and PET scanners. Hum Brain Mapp. 2014;35:1801-1814.

16. Holtbernd F, Gagnon JF, Postuma RB, et al. Abnormal metabolic network activity in REM sleep behavior disorder. Neurology. 2014;82:620-627.

17. Wu P, Yu H, Peng S, et al. Consistent abnormalities in metabolic network activity in idiopathic rapid eye movement sleep behaviour disorder. Brain. 2014;137: 3122-3128.

18. Teune LK, Renken RJ, Mudali D, et al. Validation of parkinsonian diseaserelated metabolic brain patterns. Mov Disord. 2013;28:547-551.

19. Wu P, Wang J, Peng S, et al. Metabolic brain network in the Chinese patients with Parkinson's disease based on ${ }^{18}$ F-FDG PET imaging. Parkinsonism Relat Disord. 2013;19:622-627.

20. Eckert T, Tang C, Ma Y, et al. Abnormal metabolic networks in atypical parkinsonism. Mov Disord. 2008;23:727-733.

21. Poston KL, Tang C, Eckert T, et al. Network correlates of disease severity in multiple system atrophy. Neurology. 2012;78:1237-1244.

22. Niethammer M, Tang CC, Feigin A, et al. A disease-specific metabolic brain network associated with corticobasal degeneration. Brain. 2014;137:3036-3046.

23. Hughes AJ, Ben-Shlomo Y, Daniel SE, Lees AJ. What features improve the accuracy of clinical diagnosis in Parkinson's disease: a clinicopathologic study. Neurology. 1992;42:1142-1146.

24. Gilman S, Wenning G, Low P, et al. Second consensus statement on the diagnosis of multiple system atrophy. Neurology. 2008;71:670-676.

25. Hauw JJ, Daniel SE, Dickson D, et al. Preliminary NINDS neuropathologic criteria for Steele-Richardson-Olszewski syndrome (progressive supranuclear palsy). Neurology. 1994;44:2015-2019.

26. Hughes AJ, Daniel S, Ben-Shlomo Y, Lees A. The accuracy of diagnosis of parkinsonian syndromes in a specialist movement disorder service. Brain. 2002; 125:861-870.

27. Mazziotta J, Toga A, Evans A, et al. A probabilistic atlas and reference system for the human brain: International Consortium for Brain Mapping (ICBM). Philos Trans R Soc Lond B Biol Sci. 2001;356:1293-1322.

28. Spetsieris PG, Ma Y, Dhawan V, Eidelberg D. Differential diagnosis of parkinsonian syndromes using PCA-based functional imaging features. Neuroimage. 2009;45: 1241-1252.

29. Schrag A, Ben-Shlomo Y, Quinn NP. Prevalence of progressive supranuclear palsy and multiple system atrophy: a cross-sectional study. Lancet. 1999;354:1771-1775.

30. Osaki Y, Ben-Shlomo Y, Lees AJ, et al. Accuracy of clinical diagnosis of progressive supranuclear palsy. Mov Disord. 2004;19:181-189.

31. Osaki Y, Wenning GK, Daniel SE, et al. Do published criteria improve clinical diagnostic accuracy in multiple system atrophy? Neurology. 2002;59:1486-1491.

32. Paviour DC, Price SL, Jahanshahi M, Lees AJ, Fox NC. Longitudinal MRI in progressive supranuclear palsy and multiple system atrophy: rates and regions of atrophy. Brain. 2006;129:1040-1049.

33. Reginold W, Lang AE, Marras C, Heyn C, Alharbi M, Mikulis DJ. Longitudinal quantitative MRI in multiple system atrophy and progressive supranuclear palsy. Parkinsonism Relat Disord. 2014;20:222-225.

34. Hughes AJ, Ben-Shlomo Y, Daniel SE, Lees AJ. What features improve the accuracy of clinical diagnosis in Parkinson's disease: a clinicopathologic study. 1992. Neurology. 2001;57:S34-S38.

35. Josephs KA, Petersen RC, Knopman DS, et al. Clinicopathologic analysis of frontotemporal and corticobasal degenerations and PSP. Neurology. 2006;66:41-48.

36. Mahapatra RK, Edwards MJ, Schott JM, Bhatia KP. Corticobasal degeneration. Lancet Neurol. 2004;3:736-743. 